Pacific Journal of Mathematics

SPECTRAL REPRESENTATIONS OF UNBOUNDED
NONLINEAR OPERATORS ON HILBERT SPACE 


\title{
SPECTRAL REPRESENTATIONS OF UNBOUNDED NON-LINEAR OPERATORS ON HILBERT SPACE
}

\author{
Palle E. T. Jorgensen
}

\begin{abstract}
Let $\mathcal{H}$ be a separable complex $\infty$-dimensional Hilbert space and let $\mathscr{F}$ be the Fock space of symmetric tensors over $\mathcal{H}$. We consider non-linear operators $T$ from $\mathcal{H}$ to $\mathscr{F}$ defined on a dense subspace $\mathscr{D}$ in $\mathcal{H}$ with range in $\mathscr{F}$. A symmetry and reality condition is imposed on the operators $T$ under consideration. They are generally unbounded and have different extensions $\tilde{T}$ defined on subspaces $\tilde{\mathscr{D}}$ in $\mathcal{H}$ containing $\mathscr{D}$. Generalizing a result of Arveson for bounded operators (alias functions from $\mathcal{H}$ to $\mathscr{F}$ ), we show that if $T$ is affiliated with a maximal abelian von Neumann algebra in $B(\mathcal{H})$, then it follows that there is an extension $\tilde{T}$ of $T$ which is unitarily equivalent to a (non-linear) multiplication operator.
\end{abstract}

\section{TABle OF CONTENTS}

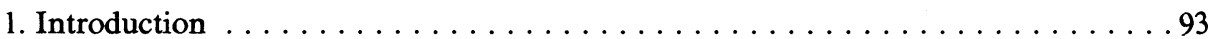

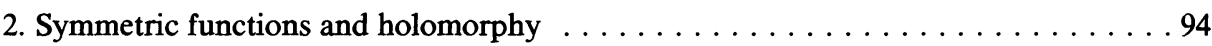

3. The extension theorem for unbounded symmetric functions $\ldots \ldots \ldots \ldots \ldots 9$

1. Introduction. Work on spectral theory of non-linear filters has been restricted so far to filters $f$ which satisfy one of several possible boundedness conditions. In the expansion into power series $f \sim\left(f_{n}\right)_{n=0}^{\infty}$, when the coefficients $f_{n}$ are sure functions, the finiteness condition $\Sigma\left\|f_{n}\right\|_{L^{2}}^{2}<\infty$ is customarily assumed (cf. [Wi] and [McK]), and this is done for important mathematical reasons. Recently, Arveson [Ar: 1,2] established a spectral theorem for non-linear "normal" processes. Here a different boundedness was assumed. The coefficients $f_{n}$ were treated as operators on the space of symmetric $n$-tensors, $n=1,2, \ldots$, and the boundedness was expressed in terms of the resulting operator norms.

The various boundedness conditions, in the literature, seem to have been dictated by the mathematical framework, rather than the applications.

In this note we consider the spectral representations of non-linear processes where the boundedness condition is dropped.

The mathematical formulation is that of [Ar: 2]. We consider non-linear functions $T$ from a given $\infty$-dimensional complex Hilbert space $\mathcal{H}$ 
into the symmetric Fock space $\mathscr{F}$ over $\mathcal{H}$. But our function $T$ is only defined on a dense linear subspace $\mathscr{D}$ in $\mathcal{H}$. Generalizing Arveson, we drop the assumption that $T$ be holomorphic (in the weak sense, i.e., for all $n=1, \ldots$, all $z_{1}, \ldots, z_{n} \in \mathscr{D}$, and $f \in \mathscr{F}$, the function

$$
a_{1}, \ldots, a_{n} \rightarrow\left(T\left(a_{1} z_{1}+\cdots+a_{n} z_{n}\right), f\right)_{\mathscr{F}} \quad \text { [inner product in } \mathscr{F} \text { ] }
$$

is entire analytic in the $n$ complex variables $a_{1}, \ldots, a_{n}$ ).

Moreover, we do not place any boundedness condition on $T$; but instead, we restrict attention to symmetric functions. The symmetry condition is expressed in terms of coherent vectors in $\mathscr{F}$. These are vectors of the form

$$
\Omega+z+2^{-1 / 2} z \otimes z+\cdots+(n !)^{-1 / 2} \overbrace{z \otimes \cdots \otimes z}^{n \text { fold }}+\cdots
$$

where $\Omega$ is the normalized basis vector in $\mathscr{F}_{0}$ (ground state), and $z$ is a fixed vector in $\mathcal{H}$. The vector in (1) is denoted by $e^{z}$, and we have

$$
\left\|e^{z}\right\|=\exp \left(\frac{1}{2}\|z\|^{2}\right) \text {. }
$$

We say that $T$ is symmetric if

$$
\left(T(z), e^{w}\right)=\left(e^{z}, T(w)\right) \text { for all } z, w \in \mathscr{D} .
$$

2. Symmetric functions and holomorphy. Arveson considered bounded holomorphic functions $T$ i.e.,

$$
\sup _{\|z\| \leq 1}\|T(z)\|<\infty \text {. }
$$

Such a function is said to be normal if it is affiliated with a maximal abelian von Neumann algebra $\mathcal{Q}$ in $B(\mathcal{H})$ (cf. [Ar: 2] for the definition of affiliation).

Multiplication operators are examples of such normal functions $T$. Let $(X, \mu)$ be a finite separable measure space, and let $t_{n}=t_{n}\left(x_{1}, \ldots, x_{n}\right)$ be a sequence of measurable symmetric functions $\left(t_{n}\right.$ on $\left.X^{n}\right)$ with a certain growth condition on $\left\|t_{n}\right\|_{\infty}$. Let $\mathscr{F}(X, \mu)$ be the symmetric Fock space over $L^{2}(X, \mu)$. Define

$$
T_{n}(z)\left(x_{1}, \ldots, x_{n}\right)=t_{n}\left(x_{1}, \ldots, x_{n}\right) z\left(x_{1}\right) \cdots z\left(x_{n}\right)
$$

for $z \in L^{2}(X, \mu)$. Then

$$
T(z)=T_{0}(z)+T_{1}(z)+\cdots
$$

is a multiplication operator. 
ThEOREM A (Arveson [Ar: 2]). Let $T: \mathcal{H} \rightarrow \mathscr{F}$ be a bounded holomorphic normal function. Then $T$ is unitarily equivalent to a multiplication operator based on some finite separable measure space; and conversely.

It follows that every $T$, satisfying the conditions in Arveson's theorem, can be expressed in the form

$$
T=F+i G
$$

where $F$ and $G$ are commuting (see below) symmetric functions from $\mathcal{H}$ to $\mathscr{F}$. Simply, let $F$ be a unitarily equivalent copy of the multiplication operator, given in (2) with $t_{n}\left(x_{1}, \ldots, x_{n}\right)$ replaced by $\operatorname{Re} t_{n}\left(x_{1}, \ldots, x_{n}\right)$, and similarly

$$
G \approx\left\{\operatorname{Im} t_{n}\left(x_{1}, \ldots, x_{n}\right)\right\} .
$$

A simple application of Segal's duality transform [Se: 1, §4] shows that every bounded symmetric function $T: \mathcal{H} \rightarrow \mathscr{F}$ extends to a symmetric (i.e., hermitian) linear operator $\tilde{T}$ in $\mathcal{F}$. However, even if $T$ is bounded, the extension $\tilde{T}$ may be unbounded.

Suppose a function $T$ from $\mathcal{H}$ to $\mathcal{F}$ can be expressed in the form $T(z)=F(z)+i G(z)$ where each of the functions $F$ and $G$ is symmetric. We then say $F$ and $G$ commute if the hermitian linear extensions $\tilde{F}$, resp. $\tilde{G}$, satisfy $(\tilde{F} f, \tilde{G} f)=(\tilde{G} f, \tilde{F} f)$ for all $f$ in the common domain.

It follows that every $T$, satisfying the conditions in Theorem A, has a decomposition of the above form.

Definition 1. A function $T: \mathcal{H} \rightarrow \mathscr{F}(\mathcal{H})$ is said to be strongly bounded if there is a bounded linear operator $\tilde{T}: \mathscr{F}(\mathcal{H}) \rightarrow \mathscr{F}(\mathcal{H})$ such that

$$
\tilde{T}\left(e^{z}\right)=T(z) \text { for all } z \in \mathcal{H} \text {. }
$$

PROPOSITION 2. Every strongly bounded function is bounded and holomorphic.

Proof. Assume $T$ is strongly bounded, and let $\tilde{T}$ be a bounded linear operator in $\mathscr{F}$ which satisfies (3). Then

$$
\sup \|T(z)\| \leq\|\tilde{T}\| \exp \left(\frac{1}{2}\right)
$$

where the supremum is taken over $z$ in $\mathcal{H},\|z\| \leq 1$. Hence $T$ is bounded. 
For $f \in \mathcal{F}$ we have $(T(z), f)=\left(\tilde{T}\left(e^{z}\right), f\right)=\left(e^{z}, \tilde{T}^{*}(f)\right)$, and the entire analytic property is clear from the following:

LEMMA 3. Let $\mathscr{F}$ be the Fock space over a given complex Hilbert space $\mathcal{H}$. Then for all $n=1,2, \ldots$, all $z_{1}, \ldots, z_{n} \in \mathcal{H}$, and all $f \in \mathcal{F}$, the function

$$
a_{1}, \ldots, a_{n} \rightarrow\left(\exp \left(\sum a_{i} z_{i}\right), f\right)
$$

is entire analytic on $\mathbf{C}^{n}$.

Proof. There are three steps in the reasoning. Consider first $f=e^{w}$ for $w \in \mathcal{H}$, second $f=$ some linear combination of coherent vectors $e^{w}$, and third $f \in \mathscr{F},\left\|f-f_{n}\right\|_{\mathscr{F}} \rightarrow 0$, where $f_{n}$ is a sequence in the algebraic linear span of the $e^{w}$ 's.

Consider now $f=e^{w}$, and $z=\sum a_{i} z_{i}$. Then

$$
\begin{aligned}
\left(e^{z}, f\right) & =\sum_{0}^{\infty}(n !)^{-1}(z \otimes \cdots \otimes z, w \otimes \cdots \otimes w)_{n}=\sum_{0}^{\infty}(n !)^{-1}(z, w)^{n} \\
& =\exp (z, w)=\exp \left(a_{1}\left(z_{1}, w\right)+\cdots+a_{n}\left(z_{n}, w\right)\right) .
\end{aligned}
$$

Hence, if $f$ is chosen as in the first and second steps, the inner product $\left(e^{z}, f\right)$ is entire analytic in the complex variables $a_{1}, \ldots, a_{n}$.

In the third step we note that if $\left\|f-f_{n}\right\|_{\mathscr{F}} \rightarrow 0$, then

$$
\left|\left(e^{z}, f_{n}\right)-\left(e^{z}, f\right)\right| \leq \exp \left(\frac{1}{2}\left\|\sum a_{i} z_{i}\right\|^{2}\right)\left\|f_{n}-f\right\| \rightarrow 0 .
$$

We have thus an approximation of the function $a_{1}, \ldots, a_{n} \rightarrow\left(e^{z}, f\right)$, given in (4), with entire analytic scalar functions, and the approximation is uniform on compact subsets of $\mathbf{C}^{n}$, i.e., the variables $a_{1}, \ldots, a_{n}$.

The lemma follows now from the Montel theorem.

At an important point in the proof of our Theorem 5 (\$3), Lemma 3 yields a corresponding automatic holomorphic property of unbounded symmetric functions ( $T$ from $\mathcal{H}$ to $\mathscr{F}$ ) which are affiliated with a maximal abelian von Neumann algebra $Q \subset \mathscr{B}(\mathcal{H})$.

EXAMPLE 4. Let $\mathcal{H}$ be a complex Hilbert space with Fock space $\mathscr{F}$. Let $A$ be a linear operator in $\mathcal{H}$ and let $\Omega(A)$ be the corresponding "quantized" operator in $\mathscr{F}$, defined in [Co, Definition 3]:

$$
\Omega(A)=\sum_{n=0}^{\infty} \sum_{i=1}^{n} A^{\delta(i, 1)} \otimes \cdots \otimes A^{\delta(i, n)},
$$

where $\delta(i, j)=1$ if $i=j$, and 0 if $i \neq j$ (we define $A^{0}$ to be the identity operators $I$ in $\mathcal{H})$. 
Then define $T_{A}(z)=\Omega(A)\left(e^{z}\right)$ for all $z$ in the domain of $A$.

(i) The function $T_{I}: \mathcal{H} \rightarrow \mathscr{F}$ is symmetric, bounded, but not strongly bounded. Moreover, $T_{I}$ is holomorphic.

(ii) Let the operator $A$ in $\mathscr{C}$ be hermitian, i.e., $(A z, w)=(z, A w)$ for all $z, w \in \mathscr{D}(A)$, the domain of $A$. Then $T_{A}$ is symmetric and, moreover, $T_{A}$ is bounded if and only if $A$ is bounded.

Proof. By [Co, Theorem 2] the operator $\Omega(A)$ is never bounded in $\mathscr{F}$ when $A \neq 0$. It is unbounded in particular when $A=I$. Note that $\Omega(I)=N$ is the number operator; indeed

$$
\Omega(I)=\sum_{n=0}^{\infty} n I^{(n)}=N .
$$

The function $z \rightarrow\left(e^{z}, f\right)$ is entire analytic on every finite-dimensional subspace $\mathscr{T C} \subset \mathcal{H}$, for all fixed vectors $f$ in $\mathscr{F}$, according to Lemma 3. Fixing $\Re$ and an orthonormal basis $z_{1}, \ldots, z_{n}$, set $z(a)=\sum a_{i} z_{i}$ and $f=e^{w}$. We may then use the chain rule on the composed mapping from

$$
a \rightarrow m=z(a): \mathbf{C}^{n} \rightarrow \text { 凡 }
$$

and

$$
m \rightarrow\left(N\left(e^{m}\right), e^{w}\right): \Re \rightarrow \mathbf{C} .
$$

Each of the two mappings is entire analytic so the chain rule applies. The analyticity of the second map is clear from the identity

$$
\left(N\left(e^{m}\right), e^{w}\right)=\sum n !^{-1} n(m, w)^{n}=(m, w) \exp ((m, w)) .
$$

The analyticity of $\left(T_{I}(z), f\right)$ for all $f \in \mathscr{F}$ now follows from the approximation argument at the end of the proof in Lemma 3. This concludes the proof of (i).

(ii) Suppose first that $T_{A}$ is bounded. Then we have

$$
\sup _{z}\left\|T_{A}(z)\right\|=M<\infty,
$$

where the supremum is taken over all $z$ in $\mathcal{H}$ such that $\|z\| \leq 1$, and $e^{z}$ is in the domain of $\Omega(A)$. We have

$$
\begin{aligned}
\left(T_{A}(z), e^{z}\right) & =\sum(n !)^{-1} n(A z, z)\|z\|^{2(n-1)} \\
& =(A z, z) \exp \left(\|z\|^{2}\right) .
\end{aligned}
$$


Hence:

$$
\begin{aligned}
|(A z, z)| & =\exp \left(-\|z\|^{2}\right)\left|\left(T_{A}(z), e^{z}\right)\right| \\
& \leq \exp \left(-\|z\|^{2}\right)\left\|T_{A}(z)\right\| \exp \left(\frac{1}{2}\|z\|^{2}\right) .
\end{aligned}
$$

Taking the supremum over $z \in \mathscr{D}(A),\|z\| \leq 1$, we get

$$
\sup _{z}|(A z, z)| \leq M \text {. }
$$

Since $A$ is hermitian, this implies boundedness of $A$, and $\|A\| \leq M$.

Assume, conversely, that $A$ is bounded. Then

$$
\begin{aligned}
\left\|T_{A}(z)\right\|^{2}= & \sum(n !)^{-1} n\|A z\|^{2}\|z\|^{2(n-1)} \\
& +\sum(n !)^{-1} n(n-1)(A z, z)^{2}\|z\|^{2(n-2)} \\
= & \left(\|A z\|^{2}+(A z, z)^{2}\right) \exp \left(\|z\|^{2}\right) .
\end{aligned}
$$

It follows that $T_{A}$ is bounded, and for the norm $M$ we have

$$
M \leq\left(2\|A\|^{2} e^{1}\right)^{1 / 2} .
$$

To check that the hermitian symmetry of $A$ implies symmetry of non-linear function $T_{A}$, a more general observation is appropriate.

Observation 1. Let $T$ be a function from $\mathcal{H}$ to $\mathscr{F}$ with domain $\mathscr{D}$, and suppose there is a hermitian symmetric operator $\tilde{T}$ in $\mathscr{F}$ such that $e^{z} \in$ $\mathscr{D}(\tilde{T})$ and $\tilde{T}\left(e^{z}\right)=T(z)$ for all $z \in \mathscr{D}$. Then it follows that $T$ is symmetric.

Proof. Obvious.

Note that the observation applies to $\tilde{T}=\Omega(A)$ for hermitian $A$, since then $\Omega(A)$ is hermitian in $\mathscr{F}$ by $[\mathbf{C o}, \S 1]$.

3. The extension theorem for unbounded symmetric functions. The verbatim parallel in the formulation of the spectral theorem for linear, and non-linear, operators (which is so striking in Arveson's theorem) is broken when the boundedness condition is dropped. Affiliation to a maximal abelian von Neumann algebra in $B(\mathcal{H C})$ is not enough to insure a spectral representation (2) when $T$ is unbounded symmetric, and non-linear, but is for unbounded linear operators, as is reflected in the interesting theorem of Stone [St]. 
TheOREM B (Stone [St]). Let $T$ be a densely defined linear operator in a complex Hilbert space $\mathcal{H}$ and assume $T$ is affiliated with a maximal abelian von Neumann algebra $\mathbb{Q}$ in $B(\mathcal{H})$.

Then it follows that $T$ is unitarily equivalent to a multiplication operator (in particular, $T$ is essentially normal, i.e., the closure $T^{-}$is a normal operator).

Our main result is an analogy to Theorem B (just as Arveson's theorem generalizes the spectral theorem for bounded linear normal operators). But for unbounded $T$, additional structure is needed for establishing a spectral representation.

We need the presence of a conjugation $J$ on $\mathcal{H}$, i.e., $J$ conjugate-linear, and satisfying $J^{2}=I$, and $(J z, w)=(J w, z), z, w \in \mathcal{H}$.

We have

TheOREM C (von Neumann [vN; RS]). Let $S$ be a hermitian symmetric linear operator which commutes with a conjugation in a Hilbert space $\mathcal{H}$. Then it follows that $S$ has selfadjoint extensions in $\mathcal{H}$.

A given conjugation $J$ on $\mathcal{H}$ clearly extends to a conjugation $\tilde{J}$ on Fock space $\mathscr{F}$ over $\mathscr{H}$ (by direct summing appropriate tensor powers of $J$ ).

We say that a function $T$ from $\mathcal{H}$ to $\mathscr{F}$ commutes with $J$ if the domain $(2)$ of $T$ is $J$-invariant and

$$
T(J z)=\tilde{J} T(z) \text { for all } z \in \mathscr{D} .
$$

THEOREM 5. Let $\mathcal{H}$ be a separable complex Hilbert space ( $\infty$-dimensional), $\mathscr{F}$ the symmetric Fock space over $\mathcal{H}, J$ a conjugation in $\mathcal{H}, T$ a symmetric function from $\mathcal{H}$ to $\mathscr{F}$ with dense domain, and $\mathscr{Q} \subset B(\mathcal{H})$ a maximal abelian von Neumann algebra. Assume

(i) $J A J=A^{*}$ for all $A \in \mathbb{Q}$.

(ii) $T$ commutes with $J$.

(iii) $T$ is affiliated with $Q$.

Then it follows that $T$ has a selfadjoint extension, unitarily equivalent to a multiplication operator, which is affiliated with $\mathbb{Q}$. There is a spectral representation (2) based on some finite separable measure space $(X, \mu)$ and on a sequence $\left\{t_{n}\left(x_{1}, \ldots, x_{n}\right)\right\}_{n=0}^{\infty}$ of measurable symmetric functions with values in the reals.

Proof. We first note that $T$ extends canonically to the linear subspace in $\mathscr{F}$ which is spanned by the (coherent) vectors $\left\{e^{z}: z \in \mathscr{D}\right\}$. We denote 
this subspace by $E(\mathscr{D})$ and note that it is dense in the Fock space $\mathscr{F}$ over $\mathcal{H C}$. Indeed, the density follows from a known ([Se 1, Theorem 3]) and easy algebraic argument coupled with the density of $\mathscr{D}$ in $\mathcal{H}$.

The following is a direct converse to Observation 1 in $\$ 2$.

Observation $1^{\prime}$. There is a unique hermitian, symmetric, linear operator $\tilde{T}$ in $\mathcal{F}$ which is defined on $E(\mathscr{D})$ and satisfies

$$
\tilde{T}\left(e^{z}\right)=T(z) \text { for } z \in \mathscr{D} \text {. }
$$

Proof. Consider a finite linear combination $f=\Sigma_{k} c_{k} e^{z_{k}}$, where $c_{k} \in \mathbf{C}$, and $z_{k} \in \mathscr{Q}$ for $k=1,2, \ldots$ If $\tilde{T}$ is given as a linear operator satisfying (5), then necessarily

$$
\tilde{T}(f)=\sum_{k} c_{k} T\left(z_{k}\right)
$$

Hence, the uniqueness!

But it is possible to define a linear operator $\tilde{T}$ via the formula (6). For if $f=\sum c_{k} e^{z_{k}}=0$, then

$$
\begin{aligned}
\left(\sum_{k} c_{k} T\left(z_{k}\right), e^{w}\right) & =\sum_{k} c_{k}\left(T\left(z_{k}\right), e^{w}\right) \\
& =\sum_{k} c_{k}\left(e^{z_{k}}, T(w)\right)=\left(\sum_{k} c_{k} e^{z_{k}}, T(w)\right) \\
& =(0, T(w))=0 \text { for all } w \in \mathscr{D} .^{1}
\end{aligned}
$$

But the vectors in $\mathscr{F}$ of the form $e^{w}$ span a dense subspace in $\mathscr{F}$, and it follows that $\tilde{T}$ is well defined through formula (6). If we consider $f=\sum c_{j} e^{z_{j}}$, and $g=\sum d_{k} e^{w_{k}}\left(c_{j}, d_{k} \in \mathbf{C}, z_{j}, w_{k} \in \mathscr{D}\right)$, then the argument used in showing that $T$ is well defined as a linear operator with domain $E(\mathscr{D})$ yields the identity

$$
(\tilde{T}(f), g)=(f, \tilde{T}(g)) .
$$

\footnotetext{
${ }^{1}$ Note that the extension argument works more generally for any function $T$ from $\mathcal{H}$ to $\mathcal{F}$ (symmetric or not) which satisfies $\left(T(z), e^{w}\right)=\left(T(J w), e^{J(z)}\right)$ for all $z, w \in \mathscr{D}$. The resulting linear extension operator $\tilde{T}$ with domain $E(\mathscr{D}) \subset \mathcal{F}$ is formally normal, and $T$ decomposes, $T=F+i G$, as a sum of commuting symmetric functions $F, G$, each commuting with $J$. If selfadjoint commuting extensions $\tilde{F}$ and $\tilde{G}$ can be found, for example by [Ne, Corollary 9.1], or [St] Theorem 21, alias Theorem B above, then the resulting joint spectral representation can be restricted to a spectral representation for $T$.
} 
Indeed, the verification reduces to the identity

$$
\left(T\left(z_{j}\right), e^{w_{k}}\right)=\left(e^{z_{\jmath}}, T\left(w_{k}\right)\right)
$$

in view of the sesquilinearity of the inner product $(\cdot, \cdot)$. Hence, the symmetry of $T$ carries over to the extension $\tilde{T}$.

Observation 2. If $U$ is a unitary operator in $\mathcal{H}$ satisfying

$$
T(U z)=\Gamma(U) T(z)
$$

for all $z \in \mathscr{D}$, then it follows that $\Gamma(U)$ commutes with $\tilde{T}$.

Proof. It is assumed that $T$ is symmetric, and $\tilde{T}$ denotes the linear hermitian symmetric extension constructed in Observation $1^{\prime}$. For $f \in$ $E(\mathscr{D}), f=\sum c_{j} e^{z_{j}}\left(c_{j} \in \mathbf{C}, z_{j} \in \mathscr{D}\right)$, we have

$$
\Gamma(U) f=\sum c_{j} \Gamma(U)\left(e^{z_{j}}\right)=\sum c_{j} e^{U\left(z_{j}\right)} .
$$

Since $U$ commutes with $T$ it follows that the domain $\mathscr{D}$ is invariant under $U$. Hence, $\Gamma(U)(f) \in E(\mathscr{D})$, and

$$
\begin{aligned}
\tilde{T} \Gamma(U)(f) & =\tilde{T}\left(\sum c_{j} e^{U\left(z_{j}\right)}\right)=\sum_{j} c_{j} T\left(U\left(z_{j}\right)\right) \\
& =\sum_{j} c_{j} \Gamma(U)\left(T\left(z_{j}\right)\right)=\Gamma(U) \tilde{T}(f) .
\end{aligned}
$$

The commutativity of the linear operators $\Gamma(U)$ and $\tilde{T}$ follows.

Since commutativity for linear operators is preserved under closure, it follows that the closure of $\tilde{T}$ commutes with $\Gamma(U)$. We shall therefore, in the sequel, use the same symbol $\tilde{T}$ for $(\tilde{T})^{-}$.

We now have commutativity of $\tilde{T}$ and $\Gamma(U)$ for all unitaries $U$ in the commutant of

$\mathbb{Q}$ in $B(\mathcal{H}): U \in \mathbb{Q}^{\prime}=\{X \in B(\mathcal{H}): X A=A X$ for all $A \in \mathbb{Q}\}$.

Since $\mathbb{Q}$ is maximal abelian, $\mathscr{Q}=\mathbb{Q}^{\prime}$.

Observation 3. There is a selfadjoint extension operator $\tilde{T}_{1}$ of $\tilde{T}$ which commutes with $\Gamma(U)$ for all unitaries $U$ in $Q$.

Proof. By general theory the given conjugation $J$ on $\mathcal{H}$ extends to a conjugation $\tilde{J}$ on $\mathscr{F}$ (by direct summing appropriate tensor powers of $J$ (cf. [Se: 1, 2])). By assumption we have

$$
T(J z)=\tilde{J} T(z) \text { for } z \in \mathscr{Q},
$$


and

$$
J A J=A^{*} \text { for } A \in \mathbb{Q} .
$$

It follows from Observation 2 and (8) that $\tilde{J}$ commutes with $\tilde{T}$. The conjugation $\tilde{J}$ restricts to an isometry of the space $\mathscr{F}_{+}=\left\{f_{+} \in \mathscr{F}_{:} \tilde{T}^{*} f_{+}\right.$ $\left.=i f_{+}\right\}$onto

$$
\mathscr{F}_{-}=\left\{f_{-} \in \mathscr{F}: \tilde{T}^{*} f_{-}=-i f_{-}\right\}
$$

the von Neumann deficiency spaces for $\tilde{T}$. Consider the closed linear subspaces $\mathscr{F}_{\mathscr{Q}}\left(f_{ \pm}\right)$generated by $\left\{\Gamma(U) f_{ \pm}: U \in \mathbb{Q}\right.$ unitary $\}$, where $f_{ \pm}$ denotes a pair of vectors in the respective spaces $\mathscr{F}_{+}$and $\mathscr{F}_{-}$. We have

$$
\tilde{J} \Gamma(U) f_{+}=\Gamma(J U J) \tilde{J} f_{+}=\Gamma\left(U^{*}\right) \tilde{J} f_{+} \in \mathscr{F}_{\mathfrak{Q}}\left(\tilde{J} f_{+}\right),
$$

and it follows that the vectors $f_{+}$and $\tilde{J} f_{+}$occur in pairs.

If $\mathscr{F}_{+}=0$, then $\tilde{T}$ is already selfadjoint and there is nothing to prove. If not, we may choose a family of normalized vectors $f_{+} \in \mathscr{F}_{+}$such that the spaces $\mathscr{F}_{\mathfrak{Q}}\left(f_{+}\right)$form a maximal orthogonal family in $\mathscr{F}_{+}$. It follows from (9) and (10) that the corresponding family $\mathscr{F}_{\mathscr{Q}}\left(\tilde{J} f_{+}\right)$is maximally orthogonal in $\mathscr{F}_{-}$. For finite linear combinations

$$
\varphi_{+}=\sum \sum c_{j k} \Gamma\left(U_{k}\right) f_{j}^{(j)}
$$

with $c_{j k} \in \mathbf{C}, U_{j} \in \mathbb{Q}^{\prime}, j=1,2, \ldots$, we may define

$$
\tilde{W}\left(\varphi_{+}\right)=\sum \sum c_{j k} \Gamma\left(U_{k}\right) \tilde{J} f_{+}^{(j)} .
$$

We claim $\tilde{W}$ is well defined as a partial isometry of the orthogonal direct sum $\Sigma^{\oplus} \mathscr{F}_{\mathbb{Q}}\left(f_{+}\right)$onto $\Sigma^{\oplus} \mathscr{F}_{\mathbb{Q}}\left(\tilde{J}_{+}\right)$, and $\tilde{W}$ commutes with $\Gamma(U)$ for all unitaries $U$ in $\mathscr{Q}$. Finally, we have $\Sigma^{\oplus} \mathscr{F}_{\mathscr{Q}}\left(f_{+}\right)=\mathscr{F}_{+}$and $\Sigma^{\oplus} \mathscr{F}_{\mathfrak{Q}}\left(\tilde{J} f_{+}\right)=\mathscr{F}_{-}$ by the maximality of the chosen family $\left\{f_{+}\right\}$. The reader is refered to [Jo] and [SI] for details of the proof at this point.

The essential step in the above argument is the following identity:

$$
\begin{aligned}
\left\|\sum \sum c_{j k} \Gamma\left(U_{k}\right) \tilde{J} f_{+}^{(j)}\right\|^{2} & =\sum \sum \sum c_{j k} \bar{c}_{j s}\left(\Gamma\left(U_{s}^{*} U_{k}\right) \tilde{J} f_{+}^{(j)}, \tilde{J f_{+}^{(j)}}\right) \\
& =\sum \sum \sum c_{j k} \bar{c}_{j s}\left(\overline{\Gamma\left(J U_{s}^{*} U_{k} J\right) f_{+}^{(j)}, f_{+}^{(j)}}\right) \\
& =\sum \sum \sum c_{j k} \bar{c}_{j s}\left(\overline{\Gamma\left(U_{k}^{*} U_{s}\right) f_{+}^{(j)}, f_{+}^{(j)}}\right) \\
& =\sum \sum \sum c_{j k} \bar{c}_{j s}\left(\overline{\Gamma\left(U_{s}\right) f_{+}^{(j)}, \Gamma\left(U_{k}\right) f_{+}^{(j)}}\right) \\
& =\left\|\sum \sum c_{j k} \Gamma\left(U_{k}\right) f_{+}^{(j)}\right\|^{2} .
\end{aligned}
$$

Commutativity of $U_{k}$ and $U_{s} \in \mathbb{Q}$ is used. 
Returning to formulas (11) and (12), we see that

$$
\left\|\tilde{W}\left(\varphi_{+}\right)\right\|=\left\|\varphi_{+}\right\| \text {. }
$$

The asserted properties of $\tilde{W}$ follow quite easily from this.

Let $\tilde{T}_{1}$ be the selfadjoint operator in $\mathscr{F}$ which extends $\tilde{T}$ and has Cayley transform

$$
\tilde{W} \oplus(\tilde{T}-i)(\tilde{T}+i)^{-1}=\tilde{W}_{1} .
$$

Then $\tilde{T}_{1}$ is the desired extension operator. It is selfadjoint since it has deficiency indices ([RS]) equal to $(0,0)$. Indeed, it is the inverse Cayley transform of the isometry $W$ with initial space, and final space, equal to $\mathscr{F}$.

Moreover, the operator $F: \mathcal{H} \rightarrow \mathcal{F}$ defined by $F(z)=\tilde{W}_{1}\left(e^{z}\right)$ satisfies the assumptions in Arveson's theorem (Theorem A). Indeed, the boundedness and the analyticity are clear from Proposition 2 and Lemma 3. For $U \in \mathbb{Q}$ (unitary) and $z \in \mathscr{D}$, we have

$$
F(U z)=\tilde{W}_{1}\left(e^{U(z)}\right)=\tilde{W}_{1} \Gamma(U)\left(e^{z}\right)=\Gamma(U) \tilde{W}_{1}\left(e^{z}\right)=\Gamma(U) F(z) .
$$

There is then, by Theorem A, a unitary isomorphism $R$ of $\mathcal{H}$ onto $L^{2}(X, \mu)$ (for some finite separable measure space $(X, \mu)$ ) such that $G=\tilde{R} F R^{*}$ is a multiplication operator. Let $G(u)=G_{0}(u)+G_{1}(u)+\cdots$ be the decomposition of $G$ in

$$
\mathscr{F}(X, \mu):=L^{2}\left(X^{0}, \mu^{0}\right) \oplus L^{2}(X, \mu) \oplus L_{s}^{2}\left(X^{2}, \mu^{2}\right) \oplus \cdots .
$$

Then

$$
G_{n}(u)\left(x_{1}, \ldots, x_{n}\right)=g_{n}\left(x_{1}, \ldots, x_{n}\right) u\left(x_{1}\right) \cdots u\left(x_{n}\right)
$$

where the function $g_{n}$ on $X^{n}$ satisfies

$$
\left|g_{n}\left(x_{1}, \ldots, x_{n}\right)\right|=1 \text { for a.e. } x_{1}, \ldots, x_{n} .
$$

The function

$$
t_{n}\left(x_{1}, \ldots, x_{n}\right)=i\left(1+g_{n}\right)\left(1-g_{n}\right)^{-1}\left(x_{1}, \ldots, x_{n}\right)
$$

is defined, a.e., in $X^{n}, n=1,2, \ldots$ Indeed, $N\left(I-\tilde{W}_{1}\right)=N\left(I-\tilde{R} \tilde{W}_{1} \tilde{R}^{*}\right)$ $=\{0\}$. From easy properties of multiplication operators, it follows that the set $\left\{\left(x_{1}, \ldots, x_{n}\right) \mid g_{n}\left(x_{1}, \ldots, x_{n}\right)=1\right\}$ has $\mu^{n}$-measure zero in $X^{n}$. As a result we note that the family of functions $\left\{t_{n}\right\}$ define a selfadjoint multiplication operator in $\mathscr{F}(X, \mu)$ which extends the symmetric operator $\tilde{R} \tilde{T} \tilde{R}^{*}$. Therefore, the original non-linear operator $T: \mathscr{Q} \rightarrow \mathscr{F}$ has an extension which is unitarily equivalent to a multiplication operator and the proof is completed. 


\section{REFERENCES}

[Ar 1] W. B. Arveson, Spectral theory for nonlinear random processes; in (p. 531-537) Symposi a Matematica-20, Instituto Nazionale di alta Matematica, Algebra $C^{*}$ e loro applicazioni in fisica teorica (1975), Academic Press, London 1976.

[Ar 2] _ A spectral theorem for non-linear operators, Bull. Amer. Math. Soc., 82 (1976), 511-513.

[Co] J. M. Cook, The mathematics of second quantization, Trans. Amer. Math. Soc., 74 (1953), 222-245.

[Jo] P. E. T. Jorgensen, Selfadjoint extension operators commuting with an algebra, Math. Zeit., 169 (1979), 41-62.

[vN] J. von Neumann, Allgemeine Eigenwerttheorie Hermitescher Funktionaloperatoren, Math. Ann., 102 (1929/30), 49-131.

[Ne] E. Nelson, Analytic vectors, Ann. Math., 70 (1959), 572-615.

[McK] McKean, Weiner's theory of non-linear noise, in (p. 191-209) Stochastic Diff. Eq., SIAM-SIAM-Proceedings, vol. 6, Providence, R.I. 1973.

[RS] M. Reed and B. Simon, Fourier analysis; Self-adjointness, Methods of Modern Math. Phys., vol. II, Academic Press, New York 1975.

[Se 1] I. E. Segal, Tensor algebras over Hilbert space I, Trans. Amer. Math. Soc., 81 (1956), 106-134.

[Se 2] _ Ergodic subgroups of the orthogonal group on a real Hilbert space, Ann. Math., 66 (1957), 297-303.

[S1] S. P. Slinker, On commuting selfadjoint extensions of unbounded operators, Indiana U. Math. J., 27 (1978), 629-636.

[St] M. H. Stone, On unbounded operators in Hilbert space, J. Indian Math. Soc., 15 (1951), 155-192.

[Wi] Wiener, Non-linear Problems in Random Theory, Wiley, New York 1958.

Received February 23, 1982.

University of Pennsylvania

Philadelphia, PA 19104-3859 


\section{PACIFIC JOURNAL OF MATHEMATICS}

EDITORS

Donald BabBITT (Managing Editor)

University of California

Los Angeles, CA 90024

Hugo Rossi

University of Utah

Salt Lake City, UT 84112

C. C. Moore and Arthur Ogus

University of California

Berkeley, CA 94720
J. DugunduI

Department of Mathematics

University of Southern California

Los Angeles, CA 90089-1113

R. FINN and H. SAMELSON

Stanford University

Stanford, CA 94305

\section{ASSOCIATE EDITORS}
R. ARENS
E. F. BECKENBACH
B. H. NeumanN
F. WolF
K. YosHIDA (1906-1982)

\section{SUPPORTING INSTITUTIONS}

UNIVERSITY OF ARIZONA

UNIVERSITY OF BRITISH COLUMBIA

CALIFORNIA INSTITUTE OF TECHNOLOGY

UNIVERSITY OF CALIFORNIA

MONTANA STATE UNIVERSITY

UNIVERSITY OF NEVADA. RENO

NEW MEXICO STATE UNIVERSITY

OREGON STATE UNIVERSITY
UNIVERSITY OF OREGON

UNIVERSITY OF SOUTHERN CALIFORNIA

STANFORD UNIVERSITY

UNIVERSITY OF HAWAII

UNIVERSITY OF TOKYO

UNIVERSITY OF UTAH

WASHINGTON STATE UNIVERSITY

UNIVERSITY OF WASHINGTON 


\section{Pacific Journal of Mathematics}

\section{Vol. 111, No. $1 \quad$ November, 1984}

Harald Brandenburg and Adam Stefan Mysior, For every Hausdorff

space $Y$ there exists a nontrivial Moore space on which all continuous

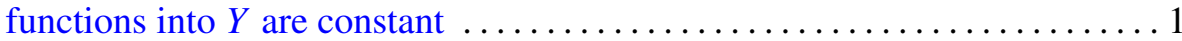

Henry Dappa, A Marcinkiewicz criterion for $L^{p}$-multipliers $\ldots \ldots \ldots \ldots \ldots 9$

P. H. Doyle, III and John Gilbert Hocking, Bijectively related spaces. I.

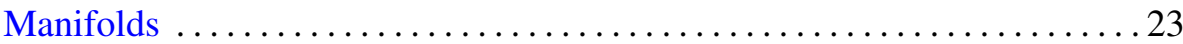

Joel Hass, Complete area minimizing minimal surfaces which are not totally

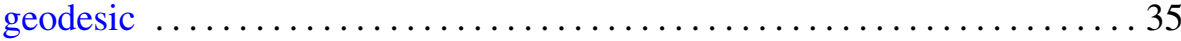

Aarno Hohti, On Ginsburg-Isbell derivatives and ranks of metric spaces .... 39

Richard Howard Hudson, Diophantine determinations of $3^{(p-1) / 8}$ and $5^{(p-1) / 4}$

A. F. Izé and A. Ventura, Asymptotic behavior of a perturbed neutral functional-differential equation related to the solution of the unperturbed

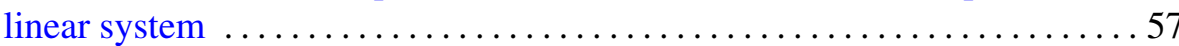

Palle E. T. Jorgensen, Spectral representations of unbounded nonlinear

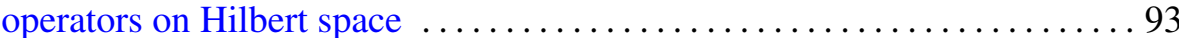

Darrell Conley Kent and Gary Douglas Richardson, Cauchy spaces with

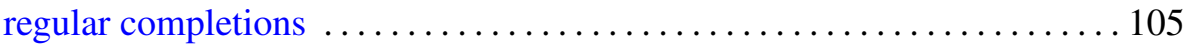

Mark Mahowald, An addendum to: "bo-resolutions" ................ 117

Stuart Wayne Margolis and Jean-Eric Pin, Minimal noncommutative varieties and power varieties

Carla Massaza and Alfio Ragusa, Some conditions on the homology groups of the Koszul complex

Vicente Miquel Molina, Some examples of Riemannian almost-product manifolds

Roderic Murufas, Inverse spectral problems for certain differential operators

Ulrich Oertel, Closed incompressible surfaces in complements of star links

Katsuro Sakai, A characterization of local equiconnectedness

William Victor Smith and Don Harrell Tucker, Weak integral convergence theorems and operator measures 